\title{
Proceso de Liquidación Técnica y Financiera de las obras por Administración Directa ejecutadas por la Gerencia Regional de Infraestructura.
}

\author{
Technical and Financial Liquidation Process of the works by Direct \\ Administration executed by the Regional Infrastructure Management.
}

Recibido: 13 de marzo del 2021

Aprobado: 22 de mayo del 2021

\section{AUTOR: \\ Mg. Quispe Huayta, Juan. Grado académico: Magister, Universidad César Vallejo, Perú. Centro laboral: Gobierno Regional de Ayacucho, ORCID: https://orcid.org/0000- 0002-0318-1041 \\ Correo: \\ johngh 10@hotmail.com}

\section{AUTOR:}

Dr. Reyes Alva, William Armando

Grado académico: Doctor, Universidad César Vallejo, Perú. Centro laboral. Universidad César Vallejo, ORCID:

https://orcid.org/00000001-9295-5987

\section{Correo:}

villyreyes@cvvirtual.edu.pe

\section{AUTOR:}

MBA. Cabrera Santa Cruz, María Julia Grado académico: MBA, Pontificia Universidad Católica del Perú. Centro laboral Universidad César Vallejos, ORCID: https://orcid.org/00000002-5361-6541

\section{Correo:}

maju2111@hotmail.com

\section{RESUMEN}

Este artículo titulado: "El proceso de liquidación técnica y financiera de las obras por Administración Directa ejecutadas por la Gerencia Regional de Infraestructura de los Gobiernos Regionales de Ayacucho e Ica en el año 2018", tiene por objetivo comprobar estadísticamente las diferencias entre el proceso de liquidación técnica y financiera de las obras ejecutadas por administración directa por las Gerencias Regionales de Infraestructura mencionadas y su importancia académica radica en ser fuente de investigación para docentes y alumnos sobre el tema. El diseño de investigación es de un modelo de tipo no experimental, con dos enfoques (cuantitativo y cualitativo) y el tipo de investigación es descriptivo comparativo. La población compuesta por los trabajos ejecutados por administración directa por las gerencias regionales de Infraestructura indicados; la muestra formada en 8 en cada una de las regiones y de 11 para la muestra piloto (6 de Ayacucho y 5 de lca). Se empleó a técnica de la encuesta y como instrumentos el cuestionario y la entrevista. Como principal conclusión se halló que no existen diferencias significativas entre el proceso de liquidación técnica y financiera de las obras ejecutadas por administración directa por la Gerencia Regional de Infraestructura en el Gobierno Regional de Ayacucho con el Gobierno Regional de Ica en el año 2018, lo cual permitirá la solución de problemática del tema en las zonas señaladas y otros. Como limitación no se halló muchos trabajos similares de los ámbitos en estudio y se apreció al revisar trabajos extranjeros la diferencia de la forma de procedimiento que se sigue para el caso de liquidación de obras, aunque el propósito es el mismo. Asimismo, los resultados hallados permiten fundamentar de manera científica, problemas de este tipo en las regiones de Ayacucho e Ica y se constituyen como aportadoras de gran contenido informativo para solución de problemas parecidos, tanto en las regiones indicadas como en otras zonas del país.

Palabras clave: Liquidación de obras, liquidación técnica, liquidación financiera, administración directa, administración pública, gobierno regional.

\section{ABSTRACT}

This article entitled "The process of technical and financial liquidation of the works by Direct Administration executed by the Regional Infrastructure Management of the Regional Governments of Ayacucho and Ica in 2018" aims to statistically verify the differences between the technical liquidation process and financial of the works executed by direct administration by the aforementioned Regional Infrastructure Managements and their academic importance lies in being a source of research for teachers and students on the subject. The research design is of a non-experimental model, with two approaches (quantitative and qualitative) and the type of research 
is comparative descriptive. The population composed of the works executed by direct administration by the indicated Regional Infrastructure Managements; the sample formed in 8 in each of the regions and 11 for the pilot sample (6 from Ayacucho and 5 from Ica). The survey technique was used and as instruments the questionnaire and the interview. As main conclusion, it was found that there are no significant differences between the process of technical and financial liquidation of the works executed by direct administration by the Regional Infrastructure Management in the Regional Government of Ayacucho with the Regional Government of Ica in 2018, which It will allow the solution of problem of the subject in the indicated zones and others. As a limitation, many similar works were not found in the areas under study and it was appreciated when reviewing foreign works the difference in the form of procedure that is followed in the case of works liquidation although the purpose is the same. Likewise, the results found allow to scientifically base problems of this type in the regions of Ayacucho and Ica and are constituted as contributors of great informative content to solve similar problems, both in the indicated regions and in other areas of the country.

Keywords: Liquidation of works, technical liquidation, financial liquidation, direct administration, public administration, regional government.

\section{1.- INTRODUCCIÓN.}

Se hace descripción de la realidad problemática sea considerando que en tiempos recientes surge la pregunta de muchas personas con respecto a las ejecuciones de trabajos públicos, sea en el contexto nacional, regional y local, como se hace y cuál es la justificación de su realización y si es importante considerando su necesidad al área usuaria. Las obras públicas son acciones desenvueltas en el rubro de la construcción de infraestructura civil que requiere de una dirección técnica, expedientes técnicos debidamente aprobados, donde están considerados la mano de obra, insumos y materiales, servicios varios, asimismo equipos necesarios para su ejecución, con débito a las cuentas de presupuesto que estén aceptadas y asignadas. Los mismos que pueden ser ejecutadas por administración directa o por modalidad de contrata y que en ambos se realizan el proceso de liquidación técnica financiera por lo que es materia de análisis de todos los factores que integran la realización a cargo de la institución, asimismo de que casi jamás se llegan a cumplir con los tiempos determinados en el cronograma del trabajo, todo esto se entiende, en desmedro del poblado beneficiario.

\section{2.- MARCO TEORICO}

Según Jiménez J. (2013) investigación cuyo Título "Gestión de contratos de obras de las administraciones públicas. Estudios de los orígenes y causas de las habituales desviaciones presupuestarias", para saber el origen de los motivos que resultan en los desvíos presupuestarios de las obras. Iglesias J. (2012) tesis titulada "Los Efectos del Proceso Penal por Delito Contra la hacienda Pública en los Procedimientos Tributarios de Liquidación y Recaudación". Lara M. (2017) tesis titulada "La Actividad Pública de Supervisión Financiera Reforma y Responsabilidad de la Administración", donde Analiza 
las consecuencias que tal reforma implica para esta actividad de control, con la finalidad de dilucidar si la regulación financiera y especialmente la bancaria, garantizar la estabilidad sistémica y los derechos de los inversores. Surge el concepto de estabilidad financiera.

Romeu J. (2018) en su tesis titulada "El principio de transparencia en la actividad contractual de la administración pública. Especial referencia a la administración local", el estudio revela que el principio de transparencia en el contexto contractual de las Administraciones Públicas españolas, Considera técnicas cualitativas y cuantitativas. Contempla la triangulación de métodos analíticos cuantitativos y cualitativos. Como conclusión se señala que el modus operandi de la administración pública se encuentra en pleno proceso de cambio. Rivera G. (2017) en su tesis titulada "Modelos y herramientas para la excelencia en la administración pública desde una perspectiva holística". El estudio es de tipo exploratorio, descriptivo y explicativo y desarrolla una investigación holística y que se complementa con la realidad concreta; 05 trabajos de investigación a nivel nacional para optar el grado de Magister: Pereyra P. (2015) en su trabajo de investigación titulada "Nivel de cumplimiento del proceso de liquidación de proyectos de inversión en el Gobierno Regional de Lima período 2015". Dicho estudio posee una dimensión cuantitativa, de modelo no experimental transversal, el modo de investigación fue básico descriptivo llegando a la conclusión que el procedimiento de liquidación se encuentra en un grado medio. Tapia Y. (2017) trabajo titulado "Evaluación de la liquidación de obras del proyecto de endeudamiento en el Gobierno Regional de Apurímac, 2013-2014". El estudio tiene como propósito medir los orígenes que interfieren el procedimiento de liquidación financiera por el modo de administración directa. Se aplicó el diseño de investigación transaccional de tipo correlacional/causal, el método utilizado fue el método descriptivo analítico y también el método deductivo. Entre las conclusiones: no se tiene un procedimiento óptimo de liquidación de obras. Sullca A. (2016) "Obras por ejecución presupuestaria directa e indirecta en las Municipalidades Provinciales de la Región Puno". La investigación permite conocer la comparación de las modalidades de realización de presupuesto directo e indirecto que realizan las municipalidades provinciales en obras de inversión pública; fue documental, descriptiva y explicativa o causal, siendo el nivel de investigación complejidad causa efecto, es decir de tipo explicativos con hipótesis de tercer nivel. Los resultados hallados del estudio permiten conocer, que la realización presupuestaria indirecta tiene mayor grado de cumplimiento en las obras, que la ejecución de presupuesto directo, en los municipios provinciales de la región Puno, durante el año 2015. Gallarday (2017) "Nivel 
de cumplimiento del proceso de liquidación de proyectos de inversión en el Gobierno Regional de Lima en el periodo del 2015”. El estudio tenía la finalidad de establecer el grado de desempeño del procedimiento de liquidación de planes de inversión por parte del Gobierno Regional de Lima durante el año 2015. Un estilo cuantitativo, cuyo diseño es no experimental y transversal, descriptivo. El procedimiento de liquidación está en un grado medio. Capcha M. (2018) "Análisis de la Eficiencia en la Ejecución de Obras Públicas por Contrata y Administración Directa en la Municipalidad Distrital de Molino Huánuco 2015". Se planteó como objetivo establecer la eficiencia en la realización de trabajos públicos por contrata y administración directa. En cuanto a los costos de ejecución final es más eficiente la ejecución por administración directa. $Y$ en cuanto a los elementos ejecutado, ninguna modalidad es más eficiente; 03 investigaciones a nivel local, de: Aguilar J. (2017) "Proceso de Liquidación Financiera y Cumplimiento de Metas y Objetivos de Obras Públicas por Administración Directa, ejecutadas en la Municipalidad Distrital de Nuñoa en el período 2012-2013". concluyendo que no efectuaron la liquidación financiera de obras públicas en forma oportuna. Uno de los factores limitantes del éxito o fracaso en el cumplir de metas y objetivos es el factor humano. Sajama S. (2018) "Liquidaciones Técnicas y Liquidaciones Financieras por Obras Ejecutadas en la Empresa Prestadora de Servicios de Saneamiento Moquegua, año 2018". El objetivo fue determinar la relación entre las liquidaciones técnicas y liquidaciones financieras por obras ejecutadas en la Empresa Prestadora de servicios de saneamiento. Guardan relación directa o indirecta con las liquidaciones de obras, siendo confirmada la hipótesis: existe relación positiva entre las liquidaciones técnicas y las liquidaciones financieras por las obras ejecutadas en la empresa prestadora de servicios de saneamiento Moquegua en al año 2018.

Se consideran en el presente estudio 08 teorías relacionadas con el tema: Existe una teoría del comportamiento que nos indica que el jefe requiere conocer las necesidades humanas para entender mejor la conducta humana, con el fin de emplear la motivación como un medio poderoso en el bienestar en la calidad de vida de los empleados como también de las instituciones (Smelser \& Suárez, 1989). Esto se puede apreciar al interno de las organizaciones de administración pública como en todo tipo de organización, por el hecho que los colaboradores manifiestan diversos tipos de necesidades y por ello diversos tipos de conducta (Garrido Luque, Ramírez Dorado, Vieira Severiano, \& Jiménez Burillo, 2013). Los colaboradores como directivos necesitan ser motivados permanentemente más aun en los organismos públicos donde prevalece la mala gestión producida en muchos casos por el desinterés de la misma institución o 
de los entes directivos a cargo. De este modo la falta de motivación o interés por los colaboradores o los planes de trabajo inciden directamente en la gestión buena o mala que presenten en cada periodo (Smelser \& Suárez, 1989). Esto es muy notorio sobre todo en los organismos de administración pública del Perú donde la mayoría de los casos se visualiza una mala gestión de sus organismos debido a la falta de interés del Estado o sus respectivos entes rectores, lo que se manifiesta entonces en los distintos comportamientos o conducta que sus colaboradores demuestran (Santacreu Mas, 2002). Hay muy poco interés por las necesidades de los colaboradores y sus expectativas tanto personales como organizacionales (Garrido Luque et al., 2013). Asimismo, según Max Weber, fue quien estableció la teoría de la burocracia como una manera de organizarse que enaltece la exactitud, la velocidad, la claridad, la regularidad y la eficiencia lograda mediante la división prefijada de las labores, de la supervisión jerárquica y de minuciosas reglas y controles (Weber, 2016).

Sin embargo, debemos aclarar que la administración pública sobre todo peruana no se caracteriza exactamente por estos criterios, sino más bien que prevalece la ineficiencia e ineficacia y por ende baja productividad de sus organizaciones (Robbins, Coulter, \& Dávila Martínez, 2005). La falta de recursos, la limitación de es estos, los malos cuadros de dirección y operativos, entre otros, son algunos de los ejemplos que se presentan en los entes de administración pública del país lo que impide tener una buena gestión tal como lo describió Weber (Desiato \& Guevara, 1998). Es así, que la doctrina de la nueva administración pública se centra en un enfoque de administración inspirado en una estructura gerencial de las organizaciones e instituciones cuyas mayores cualidades son la administración por resultados, la dirección hacia el medio, la promoción de la competitividad a, la teoría en los servicios, la calidad de la administración y un esquema de rendición de cuentas sobre los resultados (Robbins, DeCenzo, \& Mascaró Sacristán, 2002).

En el Marco conceptual se define: Las obras públicas son aquellas construcciones, edificaciones o infraestructuras que son hechas por las instituciones públicas, toda vez que posean las capacidad técnica y los requerimientos básicos para tal resultado: mano de obra, maquinaria y equipo necesario de construcción, personal técnico, dirección técnica de materiales de construcción que se necesiten para el desenvolvimiento de los trabajos respectivos, debiendo contar con toda la documentación disponible, tales como el expediente técnico y otros que aprueben su ejecución (Salinas, Álvarez y Vera ,2010). En tanto, la liquidación de obras, es vista como la acción administrativa, técnica y financiera hecha por la comisión de liquidación y recepción de obras, la misma que es nombrada por la alta dirección y cuya función es establecer de manera objetiva la 
calidad de los trabajos ejecutados en las obras. Además, señala el costo real de un proyecto u obra terminado de acuerdo con los documentos sustentatorios y estos se hallen reflejados en los registros, liquidaciones y rendiciones respectivos (Salinas et al., 2010); mientras que la liquidación financiera de obras, es la acción técnica y financiera hecha por la comisión de recepción y liquidaciones de obras públicas, según la estructura del presupuesto analítico de la obra, se toman todos los documentos fuente de los gastos reales realizados en la realización de la obra, se hace en base a los documentos contables comprobantes de pago, planillas de remuneraciones, órdenes de compra y servicio, encargos internos, cajas chicas, viáticos, nota de entrada almacén, pecosa, presupuesto analítico y sus modificaciones por específica de gastos, reporte presupuestal ejecución compromiso vs marco y otros, donde prueben el correcto empleo de los recursos recibidos y deben reflejar las inversión hecha en la ejecución de la obra o proyecto. Pues, por un lado, la liquidación técnica es el resultado técnico de obra terminada, que se confecciona con el diseño del valor referencial otorgado por el valor total realizado, que incluye los complementarios y deductivos de trabajo y cualquier otro noción generado por la variabilidad y/o cambio, que implica la variabilidad del valor de referencia; por lo que el establecimiento de los importes reales requiere una comprobación física del trabajo junto con el procedimiento de reclamación de los archivos técnicos sustentatorios de la realización del trabajo, especialmente del documento técnico y sus cambios, consolidando los documentos técnicos se procede a la contrastación o conciliación respectiva y; por otro lado, la liquidación financiera como "la reunión de acciones hechas para fijar el coste real de la realización del trabajo y el visto bueno con el presupuesto dado, por ende es un procedimiento vital y básico para comprobar la inversión hecha y el desempeño financiero, así como los documentos que lo sustentan, fijando el costo financiero auténtico del trabajo o plan realizado, que constituyen todas las salidas hechas en la cancelación de: mano de obra, materiales de construcción y otros materiales (que incluyen el empleo de saldos de inventario de otras obras y la deducción del saldo actual de almacén, instrumentos empleados y/o prestados), maquinaria y equipo (alquilado o propio) y gastos generales atribuibles a la realización del trabajo".

Pero al hablar de liquidaciones técnica y financiera, se debe mencionar a las propiedades, planta y equipo que significan los activos tangibles adquiridos 0 construidos, o en proceso de construcción, con el propósito de emplearlos de manera permanente, para la elaboración o suministro de otros bienes y servicios, para arrendarlos, o para utilizarlos en la administración del ente económico, que no están dedicados para la venta en el curso normal de los negocios y cuya vida útil excede de 
un año. (Fierro, 2009) y; también a quien lo efectiviza como es el caso del gobierno regional que representa el órgano ejecutivo y sus funciones comprenden: proponer y ejecutar el presupuesto, nombrar a los oficiales de gobierno, promulgar decretos y resoluciones, ejecutar planes y programas regionales y gerenciar las propiedades y rentas regionales. Nacen de la voluntad popular y son personas jurídicas de derecho público, con autonomía política, económica y administrativa en temas de su competencia, estableciendo para su gestión económica y financiera, un respectivo pliego presupuestal.

Según OSCE Liquidación de obras, se define en obras por contrata, como una operación técnica, realizada dentro de las condiciones plasmando en el contrato firmado (penalidades, intereses, gastos generales) con el objetivo de establecer el coste total del trabajo, los mismos que al ser comparados con los importes en pago por la institución, fijará el importe económico, que puede ser en beneficio del contratista o de la institución ejecutada (contratante). Tipos de liquidación: a) De cuentas, la liquidación de cuentas de un trabajo (acta de constatación física y su inventario en el sitio del trabajo), esto es a un trabajo no concluido producto de una resolución de contrato, que puede ser orígenes imputables al Contratista o a la Entidad Contratante. b) Final, La Liquidación final de un trabajo (acta de recepción del trabajo) se trata de una obra concluida, dentro o fuera de los plazos estipulados en el presente contrato, podemos decir con o sin penalidad. Administración Directa, proceso de ejecución de los trabajos por administración directa, esta normada por la Resolución de Contraloría №195-88CG. Es así la liquidación de las Obras por Administración Directa están reguladas por una Directiva aprobada por la autoridad competente de la Entidad Ejecutora "Directiva General Liquidación Técnica Financiera para los trabajos que se hacen por Administración directa o por encargo", concordante a la Resolución de Contraloría Nº195-88-CG, directiva donde están establecidas la mecánica operativa o el procedimiento y responsabilidades.

Las contrataciones del Estado peruano, El contrato es el acto que hacen las instituciones para abastecerse de recursos, servicios u obras, considerando la cancelación del precio o del pago que responde con dinero público, y otras obligaciones que se derivan de la situación del contratante; mientras que el contrato es el convenio para generar, regir, cambiar o eliminar un vínculo jurídico en los límites de la Ley y del Reglamento.

La investigación comprende dos dimensiones: a) Liquidación técnica: Define como el resultado técnico de obra terminada, que se confecciona con el diseño del valor referencial otorgado por el valor total realizado, que incluye los complementarios y 
deductivos de trabajo y cualquier otro noción generado por la variabilidad y/o cambio, que implica la variabilidad del valor de referencia, b)Liquidación financiera: Define como "la reunión de acciones hechas para fijar el coste real de la realización del trabajo y el visto bueno con el presupuesto dado, por ende es un procedimiento vital y básico para comprobar la inversión hecha y el desempeño financiero, así como los documentos que lo sustentan, fijando el costo financiero auténtico del trabajo o plan realizado, que constituyen todas las salidas hechas en la cancelación de: mano de obra, materiales de construcción y otros materiales (que incluyen el empleo de saldos de inventario de otras obras y la deducción del saldo actual de almacén, instrumentos empleados y/o prestados), maquinaria y equipo (alquilado o propio) y gastos generales atribuibles a la realización del trabajo".

Marco Filosófico: Si se hace un análisis profundo del significado que tiene la Administración Pública logramos identificar no sólo es una ciencia y arte sino que constituye una filosofía. Una característica saltante que presenta la Administración Pública es que tiene una esencia filosófica la cual nos dice que es servir al hombre por naturaleza, lo cual es mucho más que simplemente verla como ciencia y arte. Va más allá de solo ser arte, pues se transforma en filosofía (Chiavenato, 2002).

La formulación del problema considera: Problema general: ¿Qué diferencias existen entre el proceso de liquidación técnica y financiera de las obras ejecutadas por administración directa por la Gerencia Regional de Infraestructura en el Gobierno Regional de Ayacucho con el Gobierno Regional de Ica en el año 2018? Problemas específicos: a) ¿Qué diferencias existen en la liquidación técnica de las obras ejecutadas por administración directa por la Gerencia Regional de Infraestructura en el Gobierno Regional de Ayacucho y el Gobierno Regional de Ica en el año 2018? b) ¿Qué diferencias existen en la liquidación financiera de las obras ejecutadas por administración directa por la Gerencia Regional de Infraestructura en el Gobierno Regional de Ayacucho y el Gobierno Regional de Ica en el año $2018 ?$.

Justificación de la investigación es por: Conveniencia: Resulta conveniente investigar cuales son los elementos que se presentan en el procedimiento de liquidación de los trabajos realizados por administración directa, para poder tomar decisiones al respecto. Por Relevancia social: Con los resultados y conclusiones a obtener se conocerá cuáles son los factores presentes en el procedimiento de liquidación de los trabajos realizados por administración directa, para que de esta manera el estado pueda presentar a la sociedad una obra bien hecha, garantizando el buen uso de los recursos y al mismo tiempo complacer los requerimientos de la población. Implicaciones prácticas: Este estudio tiene utilidad práctica, porque se centra en la búsqueda de factores presentados 
en el procedimiento de liquidación de los trabajos realizados por administración directa y contribuirá en la realidad a que se pueda cumplir con la totalidad de liquidaciones de los trabajos realizados por administración directa si se pone en praxis. Valor teórico: El estudio pretende proporcionar nuevos aportes y soluciones sobre el grado de liquidaciones de los trabajos realizados por administración directa por la Gerencia Regional de Infraestructura de los Gobiernos Regionales de Ayacucho e Ica. Utilidad Metodológica: El estudio está estructurado de forma rigurosa a procedimientos establecidos, donde por medio del uso de instrumentos, se recolecta datos, y luego se procesa para poder concluir y que esto se constituya como una ayuda para futuros estudios que tengan vínculo con las liquidaciones de los trabajos ejecutados por administración directa por la Gerencia Regional de Infraestructura de los Gobiernos Regionales de Ayacucho e Ica y que sean el inicio de actividades de mejora.

Hipótesis general: Existen diferencias significativas entre el proceso de liquidación técnica y financiera de las obras ejecutadas por administración directa por la Gerencia Regional de Infraestructura en el Gobierno Regional de Ayacucho con el Gobierno Regional de Ica en el año 2018. Hipótesis nula: No existen diferencias significativas entre el proceso de liquidación técnica y financiera de las obras ejecutadas por administración directa por la Gerencia Regional de Infraestructura en el Gobierno Regional de Ayacucho con el Gobierno Regional de Ica en el año 2018. Hipótesis Específicas: HE1: Existen diferencias significativas en la liquidación técnica de las obras ejecutadas por administración directa por la Gerencia Regional de Infraestructura en el Gobierno Regional de Ayacucho y el Gobierno Regional de Ica en el año 2018. HE2: Existen diferencias significativas en la liquidación financiera de las obras ejecutadas por administración directa por la Gerencia Regional de Infraestructura en el Gobierno Regional de Ayacucho y el Gobierno Regional de Ica en el año 2018;

Objetivos: son: a) Objetivo General Cuantitativo: Comprobar estadísticamente las diferencias entre el proceso de liquidación técnica y financiera de las obras ejecutadas por administración directa por la Gerencia Regional de Infraestructura en el Gobierno Regional de Ayacucho con el Gobierno Regional de Ica en el año 2018; Objetivos Específicos Cuantitativos: OE1: Comprobar estadísticamente las diferencias en la liquidación técnica de las obras ejecutadas por administración directa por la Gerencia Regional de Infraestructura en el Gobierno Regional de Ayacucho y el Gobierno Regional de Ica en el año 2018. OE2: Comprobar estadísticamente las diferencias en la liquidación financiera de las obras ejecutadas por administración directa por la Gerencia Regional de Infraestructura en el Gobierno Regional de Ayacucho y el Gobierno Regional de Ica en el año 2018. b) Objetivo General Cualitativo: Determinar las 
diferencias en el nivel de eficacia y eficiencia en el proceso de liquidación técnica y financiera de las obras ejecutadas por administración directa por la Gerencia Regional de Infraestructura en el Gobierno Regional de Ayacucho con el Gobierno Regional de Ica en el año 2018. Objetivos Específicos Cualitativos: OE1: Determinar las diferencias en el nivel de eficacia en el proceso de liquidación técnica y financiera de las obras ejecutadas por administración directa por la Gerencia Regional de Infraestructura en el Gobierno Regional de Ayacucho con el Gobierno Regional de Ica en el año 2018. OE2: Determinar las diferencias en el nivel de eficiencia en el proceso de liquidación técnica y financiera de las obras ejecutadas por administración directa por la Gerencia Regional de Infraestructura en el Gobierno Regional de Ayacucho con el Gobierno Regional de Ica en el año 2018.

\section{3.- METODOLOGÍA}

El Diseño de investigación, la actual investigación clasifica un estudio de tipo no experimental, en cuanto identifica los factores presentes en el procedimiento de liquidación de los trabajos realizados por administración directa por la Gerencia Regional de Infraestructura de los Gobiernos Regionales de Ayacucho e Ica, donde la variable se mede sin manipulación alguna (Bravin, Pievi, Fernández Collado, \& Baptista Lucio, 2010) .

Enfoque de la investigación: Tiene dos enfoques, tanto del aporte cuantitativo en razón que para probar las hipótesis se realiza una operacionalización de la variable de origen cualitativa y se expresa en medidas las cuales si es posible cuantificar o expresar en valores numéricos sus resultados (Bravin et al., 2010). Y, por otro lado, pero complementario al estudio anterior el aporte cualitativo, a fin de poder explicar con más profundización el caso de investigación que convoca el presente trabajo (Hernández y Baptista, 2009). En cuanto al diseño, es de tipo descriptivo comparativo, diseño que como estrategia tiene como objetivo medir las diferencias que existen entre dos muestras en el Gobierno Regional de Ayacucho y en el Gobierno Regional de Ica, respecto a una variable liquidación de las obras ejecutadas sobre las que no se ha ejercido ningún control (Hernández Sampieri, Fernández Collado, \& Baptista Lucio, 2014).

La investigación consta de una sola variable que es la liquidación de Obras, definidos conceptualmente como la liquidación técnica del trabajo que se confecciona teniendo en consideración la estructuración del valor de referencia establecido por el valor ejecutado, una comprobación física del trabajo, que consiste en el procedimiento de recaudación técnica y de la realización de la liquidación financiera que es la recabacion 
de acciones para establecer el costo real de la obra; definición Operacional en la dimensión técnica ( expediente técnico y la contratación o conciliación) y la dimensión financiera (expediente financiera, considerando los indicadores (normatividad) y la escala de medición que es ordinal. Población y Muestra La población en el presente estudio fue compuesta por los trabajos ejecutados por administración directa por la Gerencia Regional de Infraestructura de los Gobiernos Regionales de Ayacucho e Ica en el año 2018. Para hacer la entrevista, la población estuvo conformada por los empleados y ejecutivos de las unidades que muestran participación en la liquidación física y financiera de los trabajos liquidados en el lapso estudiado y en las gubernaturas regionales ya mencionadas. Muestra La muestra ha sido seleccionada con el fin de lograr información respecto a los factores y la variable como es liquidación de los trabajos ejecutados por administración directa por la Gerencia Regional de Infraestructura de los Gobiernos Regionales de Ayacucho e Ica. La muestra está compuesta en la unidad de supervisión y liquidaciones de obras en cuantía de (8) en cada una de las regiones, tanto en la de Ayacucho como en la de Ica.

Técnicas e instrumentos de recolección de datos, validez y confiabilidad: Técnicas: Encuesta, para recopilar informes se hizo mediante un cuestionario previamente confeccionado, válido y confiable, sin cambiar el entorno ni el fenómeno donde se recolecta los datos de la muestra, tal y como se presentan y en un determinado momento. Estos datos se obtuvieron haciendo una reunión de preguntas normalizadas orientadas a las muestras previamente seleccionadas de las dos regiones tanto de Ayacucho como de Ica. Entrevista, se usaron con el propósito de saber estados de juicio, nociones, cualidades o eventos específicos, para esto se usó la guía de entrevista. Observación, durante el estudio o investigación se usó la observación como una técnica que permitió ver en el mismo lugar el desenvolvimiento de los hechos a investigarse y tener una opinión personal propia. Instrumentos: Cuestionario, se hicieron un formulario de preguntas preestablecidas y específicas en un número apropiado del tema a investigar. Guía de Entrevistas, Formato consistente de pautas a tenerse en cuenta durante el proceso que se realizaron durante la entrevista referente al tema a tratar. Fichas para la toma de apuntes respectivos de los aspectos más saltantes durante los procesos de observación y entrevista.

Métodos de análisis de datos, en el presente trabajo los informes que se obtenía mediante el empleo de las técnicas y herramientas confiables fueron procesados en el aplicativo de textos Word y en hojas de cálculo del aplicativo Excel. Para la estadística descriptiva, los productos se muestran en tablas de frecuencias y gráficos porcentuales con sus respectivas interpretaciones, para el trabajo del procesamiento de datos y 
presentación de tablas y figuras se usó el Software SPSS versión 24 en español. Para la estadística inferencial, se trabajó con la prueba de $U$ de Mann-Whitney, por tratarse de comparación de dos muestras independientes, respecto a una variable cualitativa. Marco Paradigmas: Para desarrollar la actual investigación se empleó el modelo hermenéutico, dado que en éste se busca la interpretación de un hecho como es determinar las causas que influyen sobre un problema planteado al cual desean darle una profunda reflexión. Los estudios centrados en administración pública corresponden entonces a la interpretación de las ciencias sociales.

\section{IV.- RESULTADOS:}

\section{Resultados Cuantitativos}

Tabla $N^{\circ} 1$. Análisis de normalidad de los datos

\begin{tabular}{|c|c|c|c|c|c|c|c|}
\hline & Gerencia & \multicolumn{3}{|c|}{ Kolmogorov-Smirnova } & \multicolumn{3}{|c|}{ Shapiro-Wilk } \\
\hline & Infraestructura & Estadístico & gl & Sig. & Estadístico & $g l$ & Sig. \\
\hline El proceso & Gerencia & ,242 & 18 & ,006 & ,867 & 18 & ,016 \\
\hline de & Regional de & & & & & & \\
\hline liquidación & Infraestructura & & & & & & \\
\hline técnica y & de Ayacucho & & & & & & \\
\hline financiera de & Gerencia & , 192 & 18 & ,079 & ,841 & 18 & ,006 \\
\hline las obras por & Regional de & & & & & & \\
\hline Administraci & Infraestructura & & & & & & \\
\hline ón Directa & de Ica & & & & & & \\
\hline
\end{tabular}

Fuente: Ordenador SPSS versión 24

En la Tabla № 1 se aprecia el estadístico de Shapiro-Wilk para el proceso de liquidación técnica y financiera de las obras por Administración Directa en la que tanto para los valores obtenidos respecto a la Gerencia Regional de Infraestructura de Ayacucho $(0,016)$ y de la Gerencia Regional de Infraestructura de Ica $(0,006)$, en ambos casos el nivel de significancia es mínimo por lo que se asume que la distribución no es normal, por lo tanto para la prueba de hipótesis se usará el estadístico no paramétrico de U de Mann-Whitney.

Objetivo general: Comprobar estadísticamente las diferencias entre el proceso de liquidación técnica y financiera de las obras ejecutadas por administración directa por la Gerencia Regional de Infraestructura en el Gobierno Regional de Ayacucho con el Gobierno Regional de Ica en el año 2018. 
Tabla $N^{\circ} 2$. Estadísticos para la puntuación obtenida respecto a las diferencias entre el proceso de liquidación técnica y financiera de las obras ejecutadas por administración directa por la Gerencia Regional de Infraestructura en el Gobierno Regional de Ayacucho con el Gobierno Regional de Ica en el año 2018.

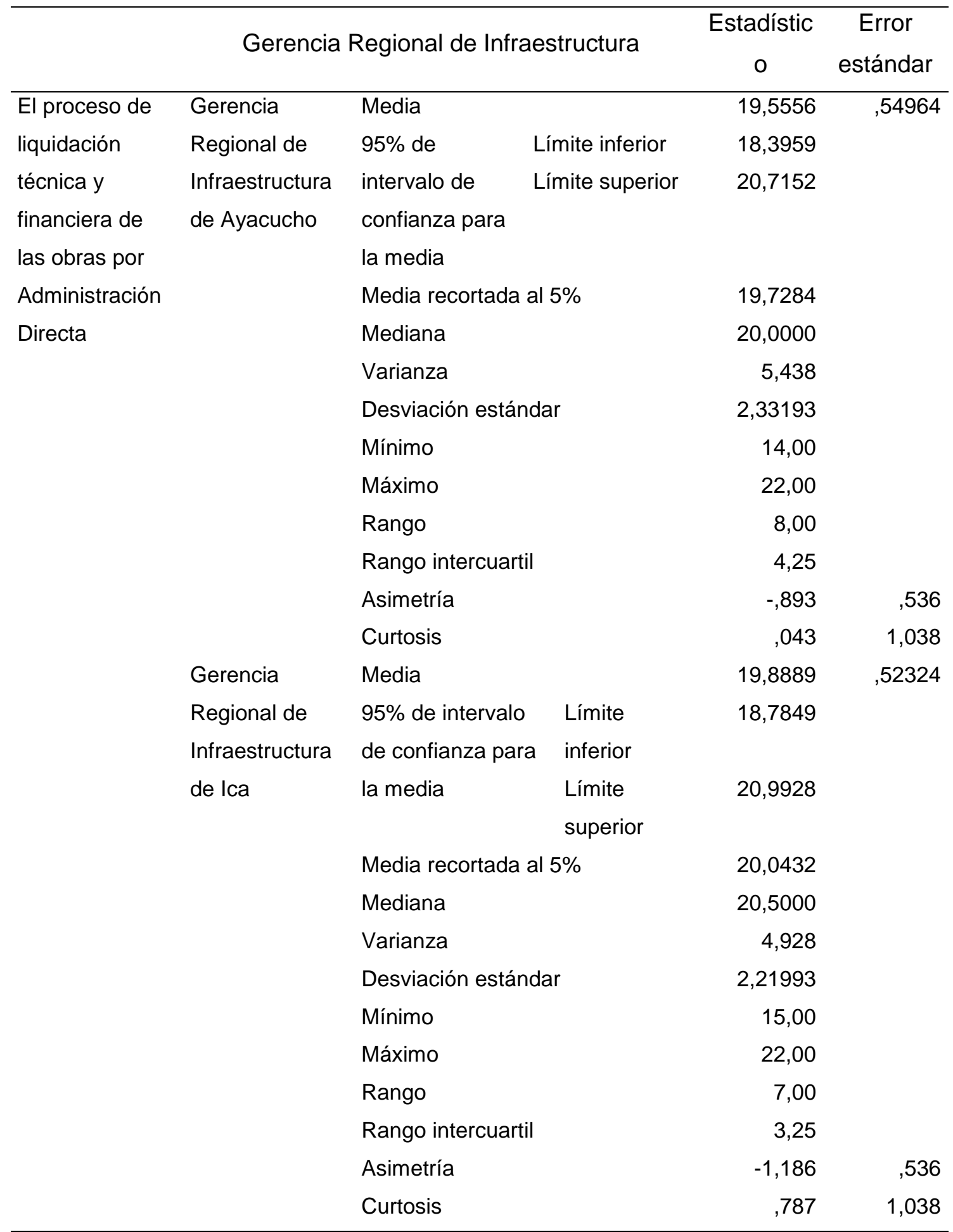

Fuente: Base de datos 
En la Tabla № 2 se encuentran los estadísticos para la puntuación obtenida respecto a las diferencias entre el proceso de liquidación técnica y financiera de las obras ejecutadas por administración directa por la Gerencia Regional de Infraestructura en el Gobierno Regional de Ayacucho en el año 2018, en la que el puntaje mínimo es 14 y el máximo 22 puntos respectivamente, con una media de 19,5556 con su intervalo de confianza con un $5 \%$ de significancia que oscila entre 18,3959 y 20,7152 .

Tabla Nº.3. Estadístico U de Mann-Whitney para la prueba de hipótesis

\begin{tabular}{lr}
\hline & $\begin{array}{r}\text { El proceso de liquidación técnica y } \\
\text { financiera de las obras por } \\
\text { Administración Directa }\end{array}$ \\
U de Mann-Whitney & 147,000 \\
W de Wilcoxon & 318,000 \\
Z & $-0,483$ \\
Sig. asintótica (bilateral) & 0,629 \\
Significación exacta [2*(sig. unilateral)] & $0,650^{\text {b }}$
\end{tabular}

a. Variable de agrupación: Gerencia Regional de Infraestructura

Fuente: Computador con SPSS versión 24 para Windows

En la Tabla $N^{\circ} 3$ se aprecia el estadístico la U de Mann-Whitney $(147,00)$ la cual asegura un $Z=-0,483$ el cual cae en una zona de aceptación de la hipótesis nula y se rechaza la hipótesis del investigador (Significancia=0,650). Se concluye que no existen diferencias significativas entre el proceso de liquidación técnica y financiera de las obras ejecutadas por administración directa por la Gerencia Regional de Infraestructura en el Gobierno Regional de Ayacucho con el Gobierno Regional de Ica en el año 2018.

Asimismo, en evaluaciones similares, en el caso de la dimensión liquidación técnica se apreció el estadístico la $U$ de Mann-Whitney $(161,00)$ la cual asegura un $Z=-0,32$ el cual cae en la zona de aceptación de la hipótesis nula y se rechaza la hipótesis del investigador (Significancia=0,988) y en el caso de la dimensión liquidación financiera se apreció el estadístico la $U$ de Mann-Whitney $(127,00)$ la cual asegura un $Z=-1,143$ el cual cae en una zona de aceptación de la hipótesis nula y se rechaza la hipótesis del investigador (Significancia=0,279).

\section{IV.- DISCUSIONES}

Los resultados alcanzados en el estudio arrojaron que se rechazó la hipótesis del investigador (Existen diferencias significativas entre el proceso de liquidación técnica y financiera de las obras ejecutadas por administración directa por la Gerencia Regional 
de Infraestructura en el Gobierno Regional de Ayacucho con el Gobierno Regional de Ica en el año 2018) y se aceptó la hipótesis nula (No existen diferencias significativas entre el proceso de liquidación técnica y financiera de las obras ejecutadas por administración directa por la Gerencia Regional de Infraestructura en el Gobierno Regional de Ayacucho con el Gobierno Regional de Ica en el año 2018). El rechazo de la hipótesis del investigador se hace efectivo con un nivel de significancia del 0,650, lo que conlleva a afirmar que entre estas dos regiones no hay una diferencia relevante entre los procesos de liquidación técnica y financiera de las obras ejecutadas por administración directa por la Gerencias Regionales de Infraestructura de Ayacucho e Ica. El factor que haga que arroje este resultado es probable que provenga por el hecho de que ambas jurisdicciones cercanas en el sur tengan la misma problemática y elementos de juicio y que corresponden a un mismo período.

Esto se evidenció por el hecho que al contrastarse de igual forma las hipótesis específicas también dieron rechazo a las formulaciones planteadas por el investigador respecto a la liquidación técnica y liquidación financiera de las gerencias regionales de infraestructura de ambos gobiernos regionales en mención; y determinan las aceptaciones de las hipótesis nulas en los dos casos. Siendo así que estos rechazos se hicieron efectivos con los niveles de significancia de: 0,988 en liquidación técnica y 0,279 en liquidación financiera.

Entre los principales aspectos se menciona: se apreció del conocimiento de la Ley No 30225 - Ley de Contrataciones del Estado y del D. S. N³50-2015, no prevalece la cultura informativa en temas de liquidación técnico-financiera en la región y se cree que en los ámbitos de educación y salud se aprecia un mejor nivel de satisfacción por ejecutar obras públicas y que el aporte más significativo de la teoría de la nueva gestión pública es la innovación del sistema público y que la burocracia está muy presente en los casos de liquidación pública, que la consecuencia principal de hacer uso eficaz y económico de los costos en obras públicas se obtendría una gestión eficaz, se dice que la idea de la interpretación filosófica de los asuntos de liquidación técnico-financiera ejecutadas está determinado por la buena gestión que debe cundir como organismo público, así como la identidad a los actos y a la organización.

\section{CONCLUSIONES:}

Conclusiones Cuantitativas. Como principal conclusión se obtuvo que no existen diferencias significativas entre el proceso de liquidación técnica y financiera de las obras ejecutadas por administración directa por la Gerencia Regional de Infraestructura en el 
Gobierno Regional de Ayacucho con el Gobierno Regional de Ica en el año 2018. Así pues no existen diferencias significativas en la liquidación técnica de las obras ejecutadas por administración directa por la Gerencia Regional de Infraestructura en el manejo de la parte técnica durante el desarrollo del proceso y periodo respectivo. Tampoco existen diferencias significativas en la liquidación financiera de las obras ejecutadas por administración directa por la Gerencia Regional en el uso de fondos públicos para la realización de las obras.

Conclusiones Cualitativas. Del estudio se despende que no existen diferencias significativas en el nivel de eficacia y eficiencia en el proceso de liquidación técnica y financiera de las obras ejecutadas por administración directa por la Gerencia Regional, así la parte gerencial es majada sin ningún contratiempo en los Gobiernos Regionales de Ayacucho e Ica. En tal sentido se evidencia que no existen diferencias significativas en el nivel de eficacia en el proceso de liquidación técnica y financiera de las obras ejecutadas por administración directa por la Gerencia Regional para alcanzar sus objetivos durante el ejercicio. Así tampoco existen diferencias significativas en el nivel de eficiencia en el proceso de liquidación técnica y financiera de las obras ejecutadas por administración directa por la Gerencia Regional con el uso adecuado u óptimo de los recursos con los que dispone la institución.

\section{REFERENCIAS}

Aoiz, M. R. (1992). El clima organizacional en la administración pública. 280. Retrieved el 5 de Agosto de 2019, from https://dialnet.unirioja.es/servlet/tesis?codigo=144669, consultado el 29/05/2019,

Arismendi Vegas, A. J. T. (2012). Gestión del talento humano como competencia GTCH- Competencia Clave para el Éxito de la Gestión del Talento en las Organizaciones. Retrieved el 7 de Agosto de 2019 from https://n9.cl/v3si consultado el 30/05/2019.

Bustamante Belaúnde, Alberto (1989). La Contratación del Estado Perú. En: Themis, № 14, (pp. 21-23). Lima, Pontificia Universidad Católica del Perú.

Chiavenato, I. (2002). Gestión del talento humano: el nuevo papel de los recursos humanos en las organizaciones. Retrieved el 25 de julio de 2019 from https://n9.cl/bq0i6 consulltado el 04/06/2019.

Chiavenato, I. (2007). Administración de recursos humanos. In McGraw-Hill / Interamericana. https://doi.org/10.1007/s13398-014-0173-7.2

Gutiérrez, L. H. S. (1995). Recursos Humanos. RAE Cases, 35(4), 72-82. https://doi.org/http://dx.doi.org/10.1590/S0034-75901995000400009, consultado el 04/06/2019. 
Hernández Sampieri, Roberto; Fernández Collado, Carlos \& Baptista Lucio, María (2014). Metodología de la Investigación, México, Mc Graw Hill, 6² Edición.

Martin Tirado, Richard (2004). El Laberinto Estatal: Historia, Evolución y Conceptos de la Contratación Administrativa en el Perú. En: Revista Arbitraje PUCP (pp. 41-77). Lima, Pontificia Universidad Católica del Perú.

Robbins, S. P., Coulter, M. K., \&DávilaMartínez, J. F. J. (2005). Administración. Retrieved from https://n9.cl/t89mk

Robbins, S. P., DeCenzo, D. A., \& Mascaró Sacristán, P. (2002). Fundamentos de administración: conceptos esenciales y aplicaciones. Retrieved from https://n9.cl/ltaq4

Rodas Ubillús, Roberto Carlo (2016). El Contrato Administrativo de Supervisión de Obra Pública (tesis de maestría). Trujillo, Universidad Nacional de Trujillo, Escuela de Posgrado.

Santacreu Mas, J. (2002). La personalidad en el marco de una teoría del comportamiento humano. Retrieved from https://n9.cl/0p62s consultado el 07/06/2019.

Sargiotti V. (2010). Definición de recursos humanos.

Smelser, N. J., \&Suárez, E. (1989). Teoría del comportamiento colectivo. Retrievedf rom https://n9.cl/axrmb consultado el 10/06/2019.

Tobon, S., \& Parra, H. (2016). La Gestión Del Talento Humano en Latinoamérica: Análisis de Algunas Experiencias (kresearch Corporation, Ed.). Retrieved from https://n9.cl/nykmz consultado el 08/06/2019.

Weber, M. (2016). ¿Qué Es La Burocracia? (2016) Create Spacelndependent Publishing Platform, Ed. Retrieved from https://n9.cl/sam3c consultado el 07/06/2019. 